\title{
AMELOBLASTOMA DA MANDÍBULA: RELATO DE DOIS CASOS*
}

\author{
Antonio Carlos Domingues de Sá1, Maurício Zardo ${ }^{2}$, Ademar José de Oliveira Paes Junior ${ }^{3}$, \\ Ricardo Pires de Souza ${ }^{4}$, Murilo Postiglioni Neme ${ }^{5}$, Ismail Sabedotti ${ }^{6}$, Ary Fernando Guimarães \\ Lovato $^{7}$, Karen Dalla Costa ${ }^{8}$, Abrão Rapoport ${ }^{9}$
}

\begin{abstract}
Resumo Ameloblastomas são neoplasias odontogênicas benignas de origem epitelial, não mineralizadas, relativamente incomuns e que apresentam um comportamento localmente agressivo. Acometem predominantemente a mandíbula e podem atingir proporções variadas, de acordo com o tempo de evolução. Radiologicamente, comportam-se como lesões císticas uniloculadas ou multiloculadas. Os achados clínicos e radiológicos auxiliam no diagnóstico diferencial, embora a avaliação histológica seja necessária para a caracterização das lesões.

Unitermos: Ameloblastoma; Mandíbula; Diagnóstico.
\end{abstract}

Abstract Ameloblastoma of the mandible: report of two cases.

Ameloblastomas are benign odontogenic neoplasms of epithelial origin, nonmineralized, relatively uncommon which present an aggressive local behavior. They affect predominantly the mandible and they can reach varied proportions depending on the evolution time. Radiographically they appear as unilocular or multilocular cystic lesions. The clinical and radiological findings assist to the differential diagnosis, although the histologic evaluation is necessary in characterization of the lesions.

Key words: Ameloblastoma; Mandible; Diagnosis.

\section{INTRODUÇÃO}

Os tumores odontogênicos são neoplasias geralmente benignas, resultantes da proliferação de células que envolvem a odontogênese.

* Trabalho desenvolvido no Serviço de Cirurgia Buco-maxilofacial do Hospital Santa Casa de Misericórdia de Ponta Grossa, Ponta Grossa, PR.

1. Especialista em Cirurgia Buco-maxilo-facial pela Universidade de São Paulo (USP) - Bauru, SP, Membro do Serviço de Cirurgia Maxilo-facial do Hospital Santa Casa de Misericórdia de Ponta Grossa, Mestrando do Curso de Pós-graduação em Ciências da Saúde do Hospital Heliópolis, São Paulo, SP, Professor da Pós-Graduação da Escola de Aperfeiçoamento Profissional da Associação Brasileira de Odontologia Ponta Grossa.

2. Doutor em Cirurgia Buco-maxilo-facial pela Universidade Estadual Paulista (Unesp) - Araçatuba, SP, Membro do Serviço de Cirurgia Maxilo-facial do Hospital Santa Casa de Misericórdia de Ponta Grossa, Coordenador da Equipe de Pós-Graduação em Cirurgia Buco-maxilo-facial da Escola de Aperfeiçoamento Profissional da Associação Brasileira de Odontologia Ponta Grossa, Professor Adjunto de Cirurgia da Universidade Estadual de Ponta Grossa.

3. Médico Residente em Radiologia e Diagnóstico por Imagem do Complexo Hospitalar Heliópolis, Mestrando do Curso de Pós-graduação em Ciências da Saúde do Hospital Heliópolis.

4. Doutor em Radiologia pela Faculdade de Medicina da USP, Professor do Curso de Pós-graduação em Ciências da Saúde do Hospital Heliópolis, Coordenador do Programa de Residência Médica em Radiologia e Diagnóstico por Imagem do Complexo Hospitalar Heliópolis.

5. Acadêmico do quarto período de Odontologia da Faculdade de Odontologia da Universidade dos Campos Gerais.

6. Médico Radiologista do Serviço de Imagem do Hospital Santa Casa de Misericórdia de Ponta Grossa.

7. Mestrando do Curso de Pós-graduação em Ciências da Saúde do Hospital Heliópolis, Professor da Disciplina de Endodontia da Pontifícia Universidade Católica do Paraná.

8. Cirurgiã-dentista, Pós-graduanda em Cirurgia Buco-maxilo-facial pela Escola de Aperfeiçoamento Profissional da Associação Brasileira de Odontologia Ponta Grossa.

9. Coordenador do Curso de Pós-graduação em Ciências da Saúde do Hospital Heliópolis.
De acordo com o padrão histológico de origem, são divididos em epiteliais, mesodérmicos e mistos ${ }^{(\mathbf{1})}$. O ameloblastoma é uma neoplasia odontogênica benigna de origem epitelial, com comportamento localmente agressivo, que representa cerca de $10 \%$ dos tumores odontogênicos ${ }^{(2)}$, afetando comumente a mandíbula $(80 \%)$ e menos freqüentemente o osso maxilar $(20 \%)^{(\mathbf{1 , 2})}$. As características de imagem do ameloblastoma assemelham-se a outras lesões mandibulares de origem odontogênica e não odontogênica. O diagnóstico é feito por exame histológico, mas os achados clínicos e estudos por imagem, através de radiografias panorâmicas e tomografia computadorizada (TC), apresentam algumas características importantes para o estreitamento do diagnóstico diferencial.

\section{RELATO DOS CASOS}

\section{Caso 1}

Paciente do sexo feminino, 33 anos de idade, branca, compareceu ao ambulatório do Serviço de Cirurgia Buco-maxilo-facial do Hospital Santa Casa de Ponta Grossa,

Endereço para correspondência: Dr. Ademar José de Oliveira Paes Junior. Rua Cônego Xavier, 276, 10 andar. São Paulo, SP, 04231-030. E-mail: ademarnet@yahoo.com

Recebido para publicação em 23/9/2003. Aceito, após revisão, em 22/12/2003.
PR, apresentando pequeno aumento de volume no terço inferior direito da face, com evolução de cerca de dois anos, sem apresentar dor. Ao exame clínico intra-oral observou-se aumento de volume na região entre os dentes canino e segundo molar, ambos do lado direito; outros sinais e sintomas estavam ausentes. Foram solicitados exames complementares de imagem (radiografias panorâmicas e TC), conforme mostram as Figuras 1A a 1D.

Ambulatorialmente, foi realizada biópsia incisional na região sinfisária e o material foi encaminhado ao laboratório de patologia da Universidade de São Paulo (USP), em Bauru, SP. O laudo descreveu o diagnóstico de ameloblastoma folicular acantomatoso.

Após o planejamento cirúrgico e solicitação dos demais exames pré-operatórios, a cirurgia para ressecção do tumor foi realizada. Sob anestesia geral e intubação nasotraqueal, foi realizada ressecção marginal do tumor, através de acesso intra-oral, com margem de segurança de $2,0 \mathrm{~cm}$. Neste caso foi preservado o feixe vásculo-nervoso. Foi fixada placa de reconstrução para preservação do leito, assim como para reforço da estrutura óssea, e realizada sutura da região abordada, conforme mostra a Figura 1E. Alta hospitalar em 48 horas após o procedimento. 


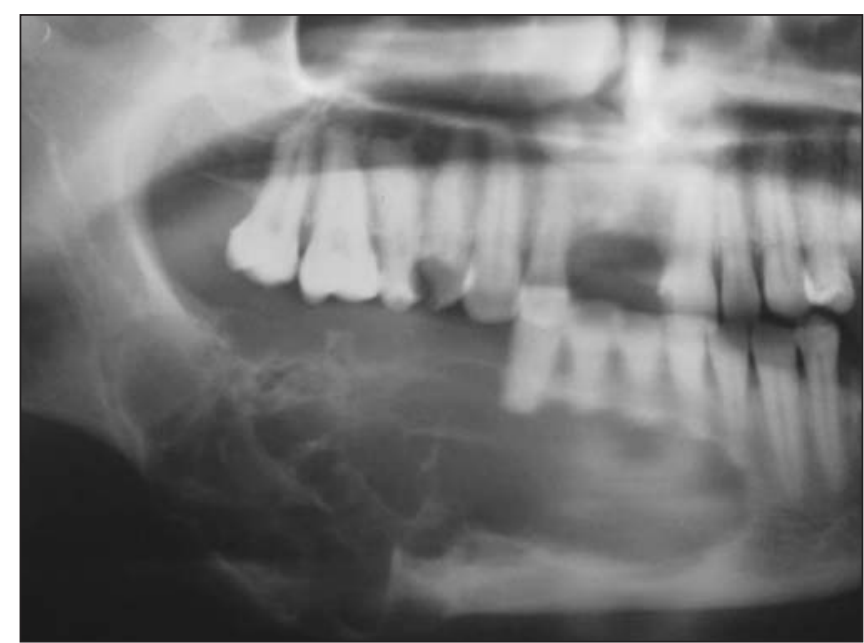

A

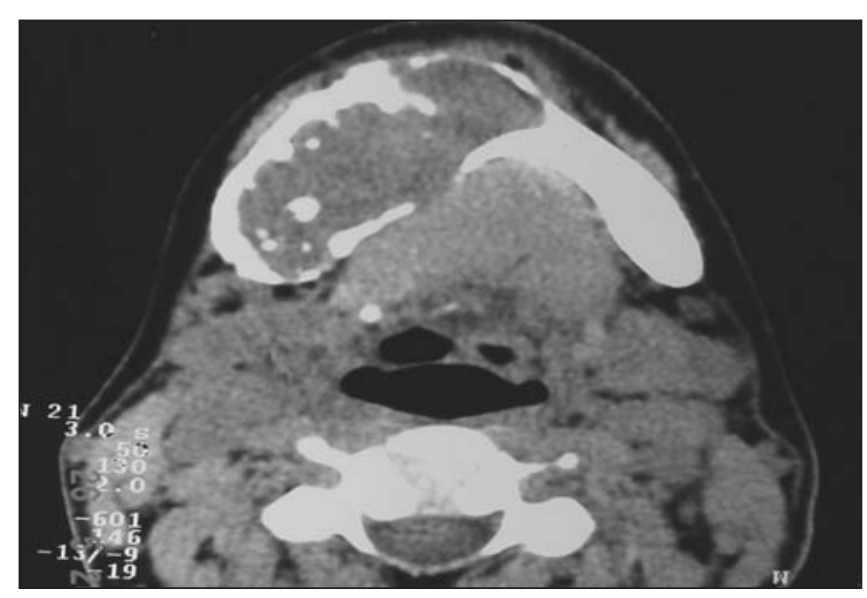

C

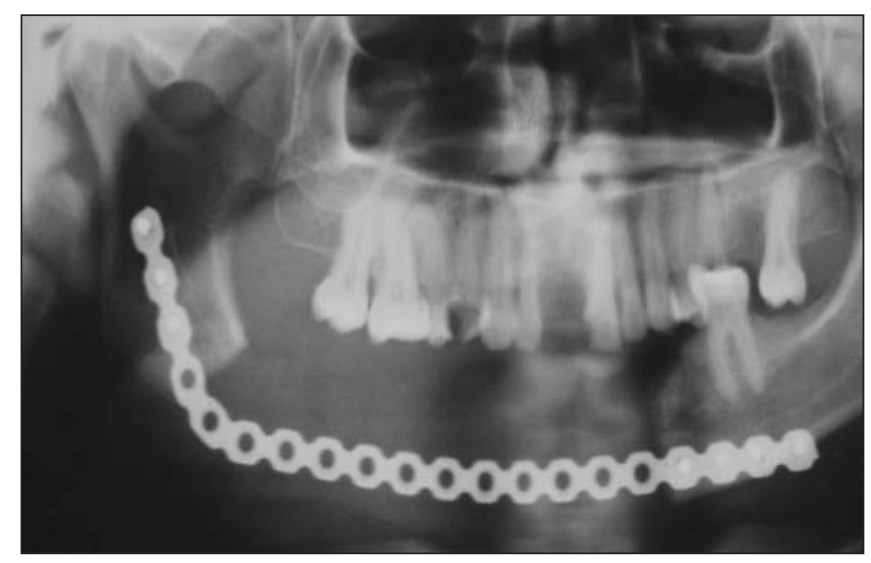

E

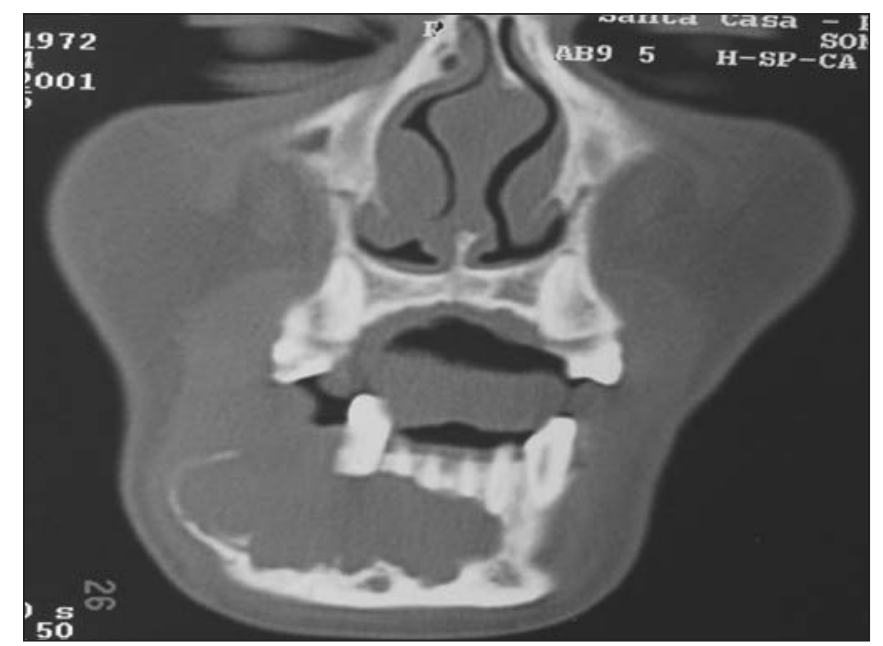

B

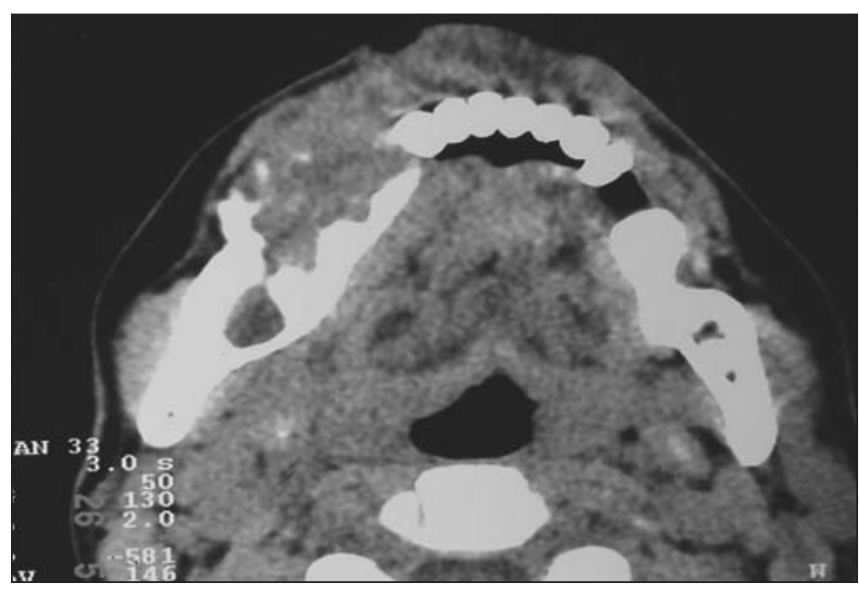

D

Figura 1. Caso 1. A: Radiografia panorâmica. Na região mentoniana, corpo e ramo horizontal direito da mandíbula, nota-se lesão lítica, insuflativa, de aspecto multiloculado, com zonas de transição parcialmente definidas, causando afilamento e áreas de rompimento da cortical óssea adjacente. Observar áreas de reabsorção das raízes de vários elementos dentais. B,C,D: Tomografia computadorizada. Cortes axiais com janela para partes moles e janela óssea demonstram lesão lítica, insuflante e multiloculada, com septos ósseos finos de permeio, densidade heterogênea, causando abaulamento e áreas de ruptura da cortical óssea das bordas lingual e alveolar, com componente de tecidos moles estendendo-se pela borda alveolar direita anteriormente. E: Radiografia panorâmica para controle pós-operatório. As imagens demonstram o aspecto pós-operatório da ressecção do tumor, com fixação de placa de reconstrução metálica.

\section{Caso 2}

Paciente de 30 anos de idade, sexo feminino, branca, compareceu ao ambulatório do Serviço de Cirurgia Buco-maxilofacial do Hospital Santa Casa de Ponta Grossa, apresentando aumento de volume na hemiface direita, inferiormente, de evolução lenta (três anos), relacionando o iní- cio do aumento de volume após um procedimento de extração dental na mesma região. Há dois meses refere dor. Não havia linfonodos cervicais palpáveis. À observação intrabucal, notou-se aumento de volume com expansão submucosa na região vestibular de todo o corpo mandibu- lar direito, desde o ângulo até a sínfise.

Após o estudo por meio de radiografia panorâmica e TC (Figuras 2A a 2D), realizou-se punção sob anestesia local, associada a biópsia incisional, cujo material obtido foi encaminhado ao laboratório de patologia da USP, em Bauru, SP. Após o 


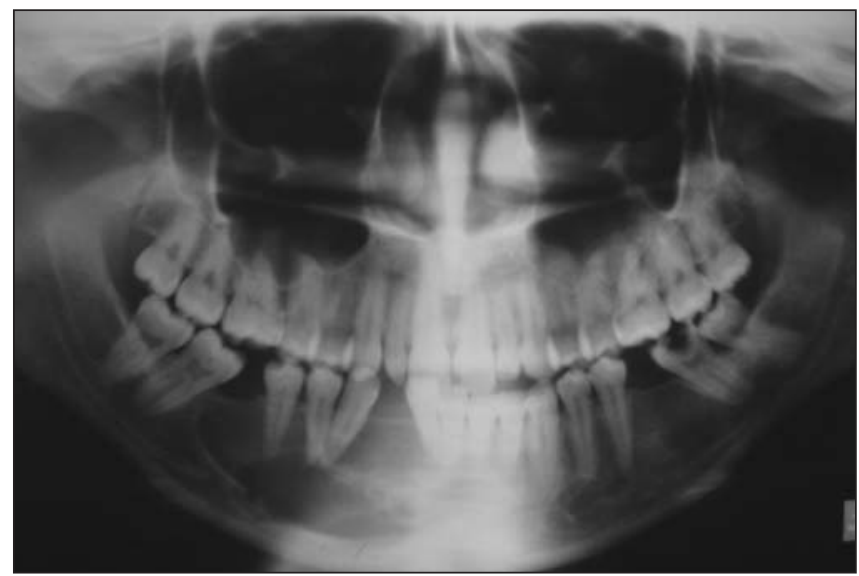

A

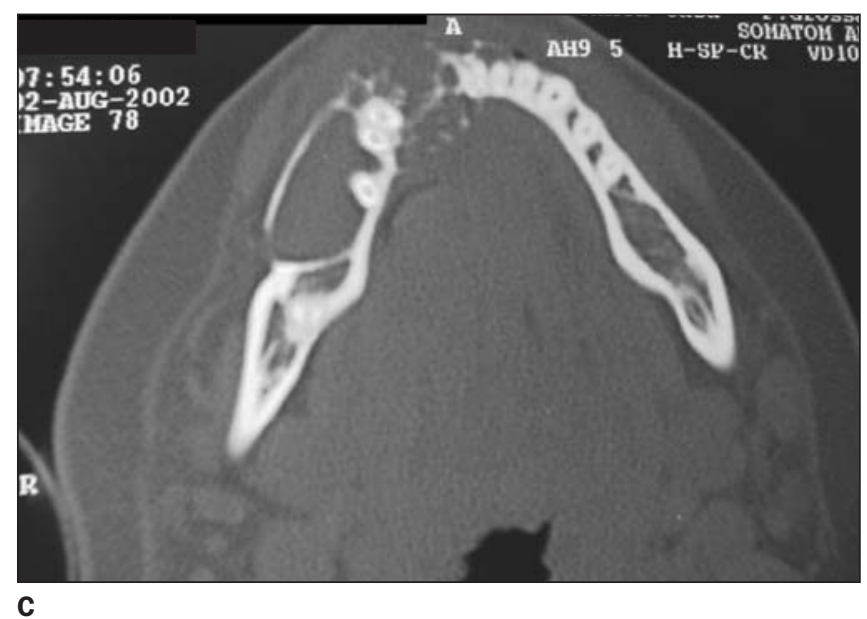

Figura 2. Caso 2. A: Radiografia panorâmica. Na região de corpo e ramo horizontal direito da mandíbula nota-se a presença de lesão radiotransparente de aspecto uniloculado, zonas de transição definidas por fina margem esclerótica, discretamente insuflativa, associada a reabsorção das raízes dentárias e deslocamento de vários dentes. Não se obsenvam áreas de rompimento da cortical óssea adjacente. B,C,D: Tomografia computadorizada. Os cortes axiais com janela para partes moles e janela óssea evidenciam lesão cística de aspecto uniloculado e discretamente insuflativa, com densidade homogênea e hipodensa em relação aos tecidos musculares, sem sinais de ruptura da cortical adjacente. E: Radiografia panorâmica para controle pós-operatório. As imagens demonstram o aspecto pós-operatório da ressecção em bloco do tumor, com fixação de placa de reconstrução metálica.

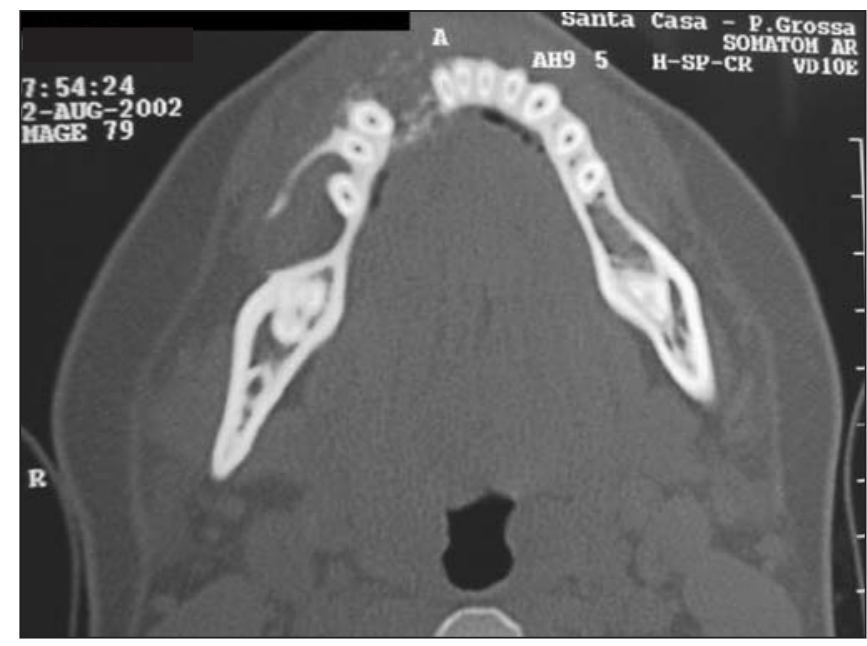

B
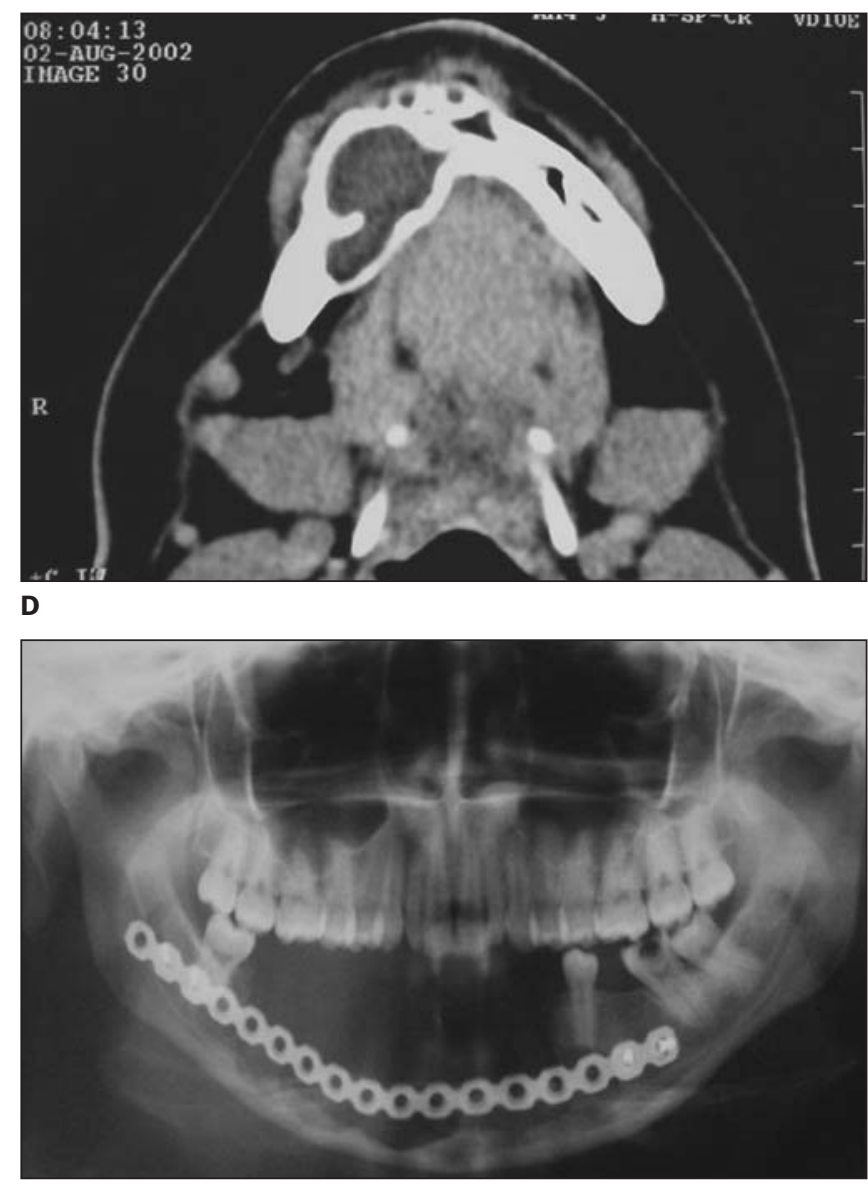

$\mathbf{E}$ diagnóstico histológico de ameloblastoma sólido folicular, foi realizada cirurgia do tipo ressecção em bloco, com margem de segurança de $2 \mathrm{~cm}$. Foi modelada placa de reconstrução e fixada para manutenção dos cotos ósseos e leito cirúrgico (Figura 2E). Alta hospitalar após 48 horas.

\section{DISCUSSÃO}

O ameloblastoma é uma neoplasia odontogênica que representa cerca de $1 \%$ de todas as neoplasias orais ${ }^{(3)}$.

O tumor é oriundo das várias camadas do epitélio odontogênico, incluindo a li- nhagem do epitélio folicular dos dentes. Manifesta-se geralmente entre a terceira e quinta décadas de vida, mas também há relatos de acometimento em pacientes de outras faixas etárias ${ }^{(\mathbf{1 , 2})}$.

A incidência entre homens e mulheres é semelhante. Apresentam-se clinicamente 
como uma massa de crescimento lento, dolorosa ou não ${ }^{(\mathbf{1})}$. A maioria dos ameloblastomas acomete os ramos e corpo posterior da mandíbula, mas os tumores mais volumosos podem infiltrar os tecidos moles adjacentes, usualmente decorrente de áreas de rompimento da cortical óssea na face lingual da mandíbula ${ }^{(2)}$.

Alvares et al. citaram o ameloblastoma como o mais agressivo dos tumores odontogênicos, devido às reabsorções radiculares, aos grandes abaulamentos e ao alto potencial de recidiva ${ }^{(4)}$.

$\mathrm{O}$ aspecto radiológico pode variar. Alguns apresentam-se como lesões radiotransparentes uniloculares bem definidas, com ou sem esclerose marginal, que, freqüentemente, estão associadas a um dente incluso; outros apresentam-se com aspecto multilocular, com septos internos e padrão em "favos de mel" ou "bolhas de sabão". As loculações podem ser ovais ou arredondadas e variam de dimensões.

São tumores caracteristicamente expansivos, podendo apresentar margens denteadas, perfurar a cortical óssea e invadir tecidos moles adjacentes. Perda da lâmina dura, reabsorção e erosão das raízes den- tárias, e deslocamento de dentes também são relatados.

Os aspectos de TC incluem áreas císticas hipodensas associadas a áreas de maior atenuação representando porções sólidas. A ressonância magnética (RM) pode, em alguns casos, demonstrar com maior clareza a extensão da lesão. Tanto a TC quanto a RM auxiliam na conduta terapêutica, delimitando o tumor ${ }^{(\mathbf{1 , 2})}$.

O comportamento do ameloblastoma tende a ser bastante agressivo nas recidivas, com maior potencial de invasão e destruição óssea do que a lesão original ${ }^{(5)}$.

Quando a lesão apresenta aspecto cístico, unilocular e bem definido, o diagnóstico diferencial se faz principalmente com ceratocistos odontogênicos, cistos dentígeros e cistos ósseos traumáticos; já o aspecto expansivo multilocular deve ser diferenciado do tumor marrom do hiperparatireoidismo, granuloma de células gigantes e hemangiomas ósseos atípicos ${ }^{(\mathbf{6})}$. A forma localmente agressiva e as formas recidivantes podem ter aspectos semelhantes aos das neoplasias malignas, devendo-se considerar o carcinoma mucoepidermóide como diagnóstico diferencial ${ }^{(2)}$.

\section{CONCLUSÃO}

Uma cuidadosa avaliação clínica e o estudo por imagem dos pacientes com lesões acometendo a mandíbula, avaliando margens da lesão, arquitetura interna, mineralização e efeitos nas estruturas adjacentes, podem auxiliar na redução do diagnóstico diferencial e na melhor conduta terapêutica para cada caso, o que é particularmente significativo nos ameloblastomas, devido à sua usual agressividade local.

\section{REFERÊNCIAS}

1. Som PM, Bergeron RT. Head and neck imaging. 3rd ed. St. Louis, MO: Mosby Year Book, 1991.

2. Scholl RJ, Kellett HM, Neumann DP, Lurie AG. Cysts and cystic lesions of the mandible: clinical and radiologic-histopathologic review. RadioGraphics 1999;19:1107-24.

3. Bataineh AB. Effect of preservation of the interior and posterior borders on recurrence of ameloblastomas of the mandible. Oral Surg Oral Med Oral Pathol Oral Radiol Endod 2000;90:155-63.

4. Alvares LC, Tavano O. Curso de radiologia em odontologia. 4르 ed. São Paulo, SP: Livraria Editora Santos, 1998.

5. Rosenstein T, Pogrel MA, Smith RA, Regezi JA. Cystic ameloblastoma: behavior and treatment of 21 cases. J Oral Maxillofac Surg 2001;59:1311-6.

6. Laskin DM, Giglio JA, Ferrer-Nuin LF. Multilocular lesion in the body of the mandible. J Oral Maxillofac Surg 2002;60:1045-8. 